\title{
Representing Sets of Orientations as Convex Cones
}

\author{
Pål Johan From and Jan Tommy Gravdahl \\ Department of Engineering Cybernetics \\ Norwegian University of Science and Technology \\ 7491 Trondheim, Norway \\ E-mail: from@itk.ntnu.no
}

\begin{abstract}
In a wide range of applications the orientation of a rigid body does not need to be restricted to one given orientation, but can be given as a continuous set of frames. We address the problem of defining such sets and to find simple tests to verify if an orientation lies within a given set. The unit quaternion is used to represent the orientation of the rigid body and we develop three different sets of orientations that can easily be described by simple constraints in quaternion space. The three sets discussed can also be described as convex cones in $\mathbb{R}^{3}$ defined by different norms. By describing the sets as convex cones and using certain properties of dual cones, we are able find simpler representations for the set of orientations and computationally faster and more accurate tests to verify if a quaternion lies within the given set.
\end{abstract}

\section{INTRODUCTION}

In certain applications, restricting the orientation of a rigid body to one frame may add unnecessary strict constraints to the modelling and control of the rigid body. Adding a freedom in the specification of the orientation is in many cases energy preserving without compromising performance and may sometimes even improve performance for a specific task. One example where energy is critical is in control of satellites. Much energy can be saved if the satellite is controlled so that the transmitter or receiver points approximately in the direction of the earth. Another example is the end effector of a robotic manipulator where an orientation error is allowed, for example in spray paint applications. The second example is motivated by the observation that a small orientation error does not affect the quality of the paint job. The speed at which the paint gun follows the path is far more critical to guarantee uniform paint coating.

In Potkonjak et al. [1] the idea of introducing the paint quality as a constraint and minimise some additional cost function was presented. This opens for the possibility of allowing an orientation error in the specifications of the end effector orientation in order to improve the performance and speed of the job, reduce torques and so on. It was shown in From and Gravdahl [2] that by allowing an orientation error in the end-effector configuration of a robotic manipulator, the speed and the quality of the job was improved. However, the orientation error was chosen intuitively, and the approach presented was not suitable for implementation in a path planning algorithm.

In From and Gravdahl [3] orientation error constraints were transformed into a test of positive definiteness of a matrix. For different types of orientation errors, a suitable matrix was found and it was shown that positive definiteness of these matrices is equivalent to an orientation that satisfies the given restrictions on the orientation. Further it was shown how to cast the restrictions on the orientation into LMIs or barrier functions.

In [3] only the size of the orientation error was restricted. The orientation error could not be restricted differently about different axes. In spray paint applications we will generally allow a bigger orientation error in the direction of the velocity, while an orientation in the transversal direction is not desired because the paint layers will overlap. There is hence a need to be able to also restrict orientation errors in different directions differently. This could not be achieved by the ice-cream cone shaped set of orientations presented in [3].

We present two different sets of orientations that allow us to restrict the orientation errors about the axes directly. The restrictions that define the desired set cannot be represented by simple constraints on the quaternion directly, as with the ice-cream cone one. This comes from the simple fact that rotations are not commutative so when more than one rotation is required to describe the volume it cannot be described by simple restrictions on the Euler angles.

We choose to represent the set of orientations as a convex set. The convex cone that restricts the rotations about the axes directly is defined by the $\infty$-norm. To find this, we first find the convex cone defined by the 1-norm and then use the property that the cone defined by the $\infty$-norm is the dual of the 1-norm to find the desired cone. This removes the discontinuity and non-differentiability of the direct definition of the $\infty$-norm. A smooth differentiable function is desirable when introducing this freedom in an optimisation algorithm to find the optimal orientation at each time step.

\section{REPRESENTING ROTATIONS}

Most of the fundamental principles of rotation were presented in two papers by Leonhard Euler in 1775 [4]. The first paper shows that any rotation can be accomplished by a sequence of three rotations about the coordinate axes. In the second paper, Euler states that any orientation can be represented by a rotation of some angle $\phi$ about some fixed axis $\boldsymbol{n}$. He also shows that the composition of two rotations is itself a rotation. 


\section{A. The Unit Quaternion}

The unit quaternion representation closely relates to the results presented in Euler's second paper. A good introduction to quaternions is found in [5]. Any positive rotation $\phi$ about a fixed unit vector $\boldsymbol{n}$ can be represented by the four-tuple

$$
Q=\left[\begin{array}{c}
q_{0} \\
\boldsymbol{q}
\end{array}\right]
$$

where $q_{0} \in \mathbb{R}$ is known as the scalar part and $\boldsymbol{q} \in \mathbb{R}^{3}$ as the vector part. $Q(\phi, \boldsymbol{n})$ is written in terms of $\phi$ and $\boldsymbol{n}$ by

$$
q_{0}=\cos \left(\frac{\phi}{2}\right), \quad \boldsymbol{q}=\sin \left(\frac{\phi}{2}\right) \boldsymbol{n} .
$$

$Q$ is a quaternion of unit length and denoted a unit quaternion. Henceforth, all quaternions have unit length if not other is stated. Let $Q_{P}=\left[\begin{array}{ll}p_{0} & \boldsymbol{p}^{\top}\end{array}\right]^{\top}$. A multiplication of two quaternions is given by a quaternion product and is written in vector algebra notations as

$$
Q_{P} * Q=\left[\begin{array}{c}
p_{0} q_{0}-\boldsymbol{p} \cdot \boldsymbol{q} \\
p_{0} \boldsymbol{q}+q_{0} \boldsymbol{p}+\boldsymbol{p} \times \boldsymbol{q}
\end{array}\right] .
$$

The cross product implies that quaternion multiplication is not commutative, as expected. Let $Q_{P}=$ $\left[\begin{array}{llll}p_{0} & p_{1} & p_{2} & p_{3}\end{array}\right]^{\top}$ and $Q=\left[\begin{array}{llll}q_{0} & q_{1} & q_{2} & q_{3}\end{array}\right]^{\top}$. Then the quaternion product is written as

$$
Q_{P} * Q=\left[\begin{array}{l}
p_{0} q_{0}-p_{1} q_{1}-p_{2} q_{2}-p_{3} q_{3} \\
p_{0} q_{1}+p_{1} q_{0}+p_{2} q_{3}-p_{3} q_{2} \\
p_{0} q_{2}+p_{2} q_{0}+p_{3} q_{1}-p_{1} q_{3} \\
p_{0} q_{3}+p_{3} q_{0}+p_{1} q_{2}-p_{2} q_{1}
\end{array}\right] .
$$

The quaternion product of two unit quaternions is a unit quaternion. By the definition of the quaternion the quaternions $Q$ and $-Q$ produce the same rotation. This is referred to as the dual covering. The quaternion identity is given by $Q_{I}=\left[\begin{array}{llll}1 & 0 & 0 & 0\end{array}\right]^{\top}$.

A pure quaternion is a quaternion with zero scalar part. Any vector, $\overline{\boldsymbol{v}}=\left[\begin{array}{lll}x & y & z\end{array}\right]^{\top}$ can be represented by a pure quaternion

$$
\boldsymbol{v}=\left[\begin{array}{l}
0 \\
\overline{\boldsymbol{v}}
\end{array}\right] \text {. }
$$

The conjugate of a quaternion is defined as

$$
Q^{*}=\left[\begin{array}{llll}
q_{0} & -q_{1} & -q_{2} & -q_{3}
\end{array}\right]^{\top} .
$$

\section{B. Quaternions and Rotations}

Let a vector, $\overline{\boldsymbol{v}}_{1}$, be represented by the pure quaternion $\boldsymbol{v}_{1}$. This vector can be rotated $\phi$ radians around the axis $\boldsymbol{n}$ by

$$
\boldsymbol{v}_{2}=Q * \boldsymbol{v}_{1} * Q^{*}
$$

Every vector $\overline{\boldsymbol{v}} \in \mathbb{R}^{3}$ can be represented by a pure quaternion, hence $\boldsymbol{v}$ is not necessarily a unit quaternion. The quaternion, $Q(\phi, \boldsymbol{n})$, however, is unitary. This represents the angle and the axis that the vector $\overline{\boldsymbol{v}}_{1}$ is to be rotated about. The resulting vector, $\overline{\boldsymbol{v}}_{2}$, is then of the same length as $\overline{\boldsymbol{v}}_{1}$ if and only if $Q$ is a unit quaternion. The quaternion representation also leads to a useful formula for finding the shortest rotation from one orientation to another. Let $Q_{P}$ and $Q$ be two orientations. Then, by taking

$$
E=Q_{P}^{*} * Q,
$$

$E$ will rotate $Q_{P}$ into $Q$ by the shortest rotation.

Note that Equation (8) rotates one frame into another frame. By a frame it is meant a coordinate system in $\mathbb{R}^{3}$ using Cartesian coordinates. One frame with respect to another frame represents three degrees of freedom and is referred to as an orientation. The inertial frame is denoted, $\mathcal{F}_{I}$ and the frame that correspond to the inertial frame by a rotation $Q$ from the inertial frame is denoted $\mathcal{F}_{Q}$. Equation (7) rotates one vector into another vector and has two degrees of freedom (e.g. longitude and latitude) [6]. A unit vector with respect to a unit reference vector is referred to as a direction. Henceforth, the main concern is with the direction of the central axis, which is assumed to be the body frame $z$-axis of the end effector.

\section{Rotation Sequences}

In this paper, the orientation is represented by a rotation sequence of three rotations about the unitary axes. We will consider two different sequences that both give a complete description of the orientation.

1) The ZYZ-sequence: The ZYZ-sequence is given by first a rotation $\alpha$ about the $z$-axis followed by a rotation $\beta$ about the new $y$-axis. This describes the direction of the $z$-axis. The last degree of freedom is given by the rotation $\gamma$ about the $z$-axis. When the sequence is given, a one-to-one mapping ${ }^{1}$ between $(\alpha, \beta, \gamma)$ and the quaternion $Q=\left[\begin{array}{llll}q_{0} & q_{1} & q_{2} & q_{3}\end{array}\right]^{\top}$ can be found whenever $\beta \neq 0$.

Given a quaternion $Q$. Then $\alpha, \beta$ and $\gamma$ from the ZYZsequence are found by [8]

$$
\begin{gathered}
\alpha=\arctan 2\left(\frac{q_{2} q_{3}-q_{0} q_{1}}{q_{0} q_{2}+q_{1} q_{3}}\right), \\
\beta=2 \arcsin \sqrt{q_{1}^{2}+q_{2}^{2}}, \\
\gamma=\arctan 2\left(\frac{q_{2} q_{3}+q_{0} q_{1}}{q_{0} q_{2}-q_{1} q_{3}}\right) .
\end{gathered}
$$

Assume that we would like to restrict the $z$-axis of $\mathcal{F}_{Q}$ to point in approximately the same direction of the $z$-axis of the inertial frame $\mathcal{F}_{I}$. This can be visualised by a cone of directions and restricted by $|\beta| \leq \beta_{\text {lim }}$ where $0 \leq \beta_{\text {lim }} \leq \pi$. The orientation error $\beta$ can be found from $q_{1}$ and $q_{2}$ from (10) directly.

2) The XYZ-sequence: The XYZ-sequence is given by first a rotation $\alpha$ about the $x$-axis followed by a rotation $\beta$ about the new $y$-axis. This describes the direction of the $z$-axis. The last degree of freedom is given by the rotation $\gamma$ about the $z$-axis. We see that the XYZ-sequence allows us to restrict the orientation about the $x$ - and $y$ axes independently through $\alpha$ and $\beta$, which is what we wanted. This cannot be achieved by that ZYZ-sequence. In

\footnotetext{
${ }^{1}$ If the dual covering of the quaternion is taken into account, a one-to-two mapping can be found.
} 
[8] simple expressions for the rotations $\alpha, \beta$ and $\gamma$ were presented for the ZYZ-sequence by reducing the equations of the resulting quaternion. Unfortunately, the equations for the XYZ-sequence do not reduce in the same way. We thus propose to the reduce the equations of the rotated vector $e_{z}=\left[\begin{array}{lll}0 & 0 & 1\end{array}\right]^{\top}$ to find similar expressions. This is shown in Section III.

\section{Convex Cones and Duality}

We will use the definition and notation found in [7] and define a set $\mathcal{C}$ a cone if for every $x \in \mathcal{C}$ and $\theta \geq 0$ we have $\theta x \in \mathcal{C}$. The set $\mathcal{C}$ is a convex cone if for any $x_{1}, x_{2} \in \mathcal{C}$ and $\theta_{1}, \theta_{2} \geq 0$ we have

$$
\theta_{1} x_{1}+\theta_{2} x_{2} \in \mathcal{C}
$$

We will denote a cone by $K$. Then, the dual cone $K^{*}$ is defined by

$$
K^{*}=\left\{y \mid x^{\top} y \geq 0 \text { for all } x \in K\right\} .
$$

The dual cone $K^{*}$ is always convex, even if $K$ is not. $K^{*}$ is also closed and we have $K^{* *}=K$.

\section{E. Convex Cones in $\mathbb{R}^{3}$}

We consider three different cones in $\mathbb{R}^{3}$ given in Figure 1 . We are mainly concerned with the direction of the $z$-axis in the rotated frame with respect to the $z$-axis of $\mathcal{F}_{I}$. The maximum rotation allowed by the cone is thus given by (the $\|\cdot\|_{2}$-norm)

$$
\beta_{\text {lim }}=\arctan \frac{\sqrt{x^{2}+y^{2}}}{|z|} .
$$

The cones are defined by the degree of the norm, representing the shape of the cone, and by a parameter $\xi$ representing the size of the cone by

$$
\left\|x_{1}, x_{2}, \ldots, x_{n-1}\right\| \leq \xi\left|x_{n}\right| .
$$

As we are mainly concerned with the $z$-axis in $\mathbb{R}^{3}$ we write

$$
\|x, y\| \leq \xi|z| .
$$

The following lemmas are useful when it comes to representing an orientation error or maximum rotation as a cone.

Lemma 2.1: Given a $\|\cdot\|_{2}$-cone with the parameter $\nu$ restricting the direction of the $z$-axis, i.e.

$$
\sqrt{x^{2}+y^{2}} \leq \nu|z| \text {. }
$$

Then the maximum rotation allowed by this cone is

$$
\beta_{\text {lim }}=\arctan \nu
$$

around any axis in the $x y$-plane. This is obtained by the ZYZ-sequence and can be visualised in Figure 1a).

Proof: The limit value is given by Equation (17) with equality. The maximum allowed rotation is given simply by the inverse tangent of the $\|\cdot\|_{2}$-norm displacement in the $x y$ plane over $z$ :

$$
\begin{aligned}
\beta_{\text {lim }} & =\arctan \frac{\sqrt{x^{2}+y^{2}}}{|z|} \\
& =\arctan \nu .
\end{aligned}
$$
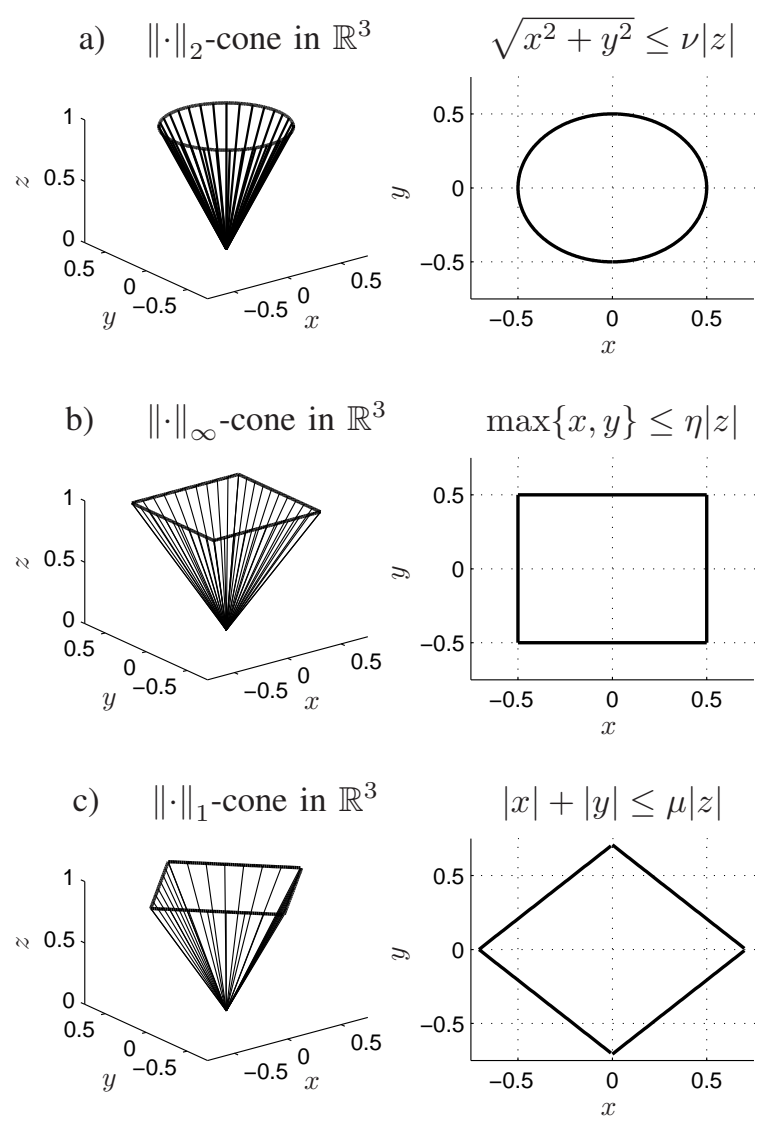

Fig. 1. Different convex cones in $\mathbb{R}^{3}$. The cone defined by the 2-norm is self dual (setting $\mu=1$ ). The cone defined by the $\infty$-norm is the dual of the cone defined by the 1-norm.

Lemma 2.2: Given a $\|\cdot\|_{\infty}$-cone with the parameter $\eta$ restricting the direction of the $z$-axis, i.e.

$$
\max \{x, y\} \leq \eta|z| .
$$

Then the maximum rotation allowed by this cone is

$$
\beta_{\text {lim }}=\arctan \eta
$$

around the principal axes ( $x$ - and $y$-axes) and

$$
\beta_{\text {lim }}=\arctan \sqrt{2} \eta
$$

around the axes $x= \pm y$. This is obtained by the XYZsequence and can be visualised in Figure 1b).

Proof: In the direction of the principal axes, the maximum possible displacement is found simply by setting $y=0$ and we get

$$
|x| \leq \eta|z|
$$

which gives us

$$
\beta_{\text {lim }}=\arctan \eta
$$


When $x= \pm y$ we have

$$
\left\|\left[\begin{array}{ll}
x & y
\end{array}\right]\right\|_{2}=\sqrt{x^{2}+y^{2}}=\sqrt{2}|x|
$$

which gives us

$$
\begin{aligned}
\beta_{\text {lim }} & =\arctan \frac{\sqrt{2}|x|}{|z|} \\
& =\arctan \sqrt{2} \eta .
\end{aligned}
$$

Lemma 2.3: Given a $\|\cdot\|_{1}$-cone with the parameter $\mu$ restricting the direction of the $z$-axis, i.e.

$$
|x|+|y| \leq \mu|z|
$$

Then the maximum rotation allowed by this cone is

$$
\beta_{\text {lim }}=\arctan \mu
$$

around the principal axes ( $x$ - and $y$-axes) and

$$
\beta_{\text {lim }}=\arctan \frac{\mu}{\sqrt{2}}
$$

around the axes $x= \pm y$. This is the dual of the $\|\cdot\|_{\infty}$-cone and can be visualised in Figure 1c).

Proof: In the direction of the principal axes, the maximum possible displacement is found simply by setting $y=0$ and we get

$$
|x| \leq \mu|z|
$$

which gives us

$$
\begin{aligned}
\beta_{\text {lim }} & =\arctan \frac{|x|}{|z|} \\
& =\arctan \mu .
\end{aligned}
$$

When $x= \pm y$ we have

$$
\left\|\left[\begin{array}{ll}
x & y
\end{array}\right]\right\|_{2}=\sqrt{\left(\frac{1}{2} x\right)^{2}+\left(\frac{1}{2} y\right)^{2}}=\frac{1}{\sqrt{2}}|x|
$$

which gives us

$$
\begin{aligned}
\beta_{\text {lim }} & =\arctan \frac{\frac{1}{\sqrt{2}}|x|}{|z|} \\
& =\arctan \frac{\mu}{\sqrt{2}} .
\end{aligned}
$$

Note that the maximum rotation for the $\|\cdot\|_{1}$-cone is given by $\arctan \mu$ while for the $\|\cdot\|_{\infty}$-cone it is given by $\arctan \sqrt{2} \eta$

For a vector $v \in \mathbb{R}^{3}$ that satisfies the restrictions given by the cone $K$ we will write $v \in K$. Similarly for a quaternion $Q$ we will write $Q \in K$ if the vector that results from the rotation of the vector $e_{z}=\left[\begin{array}{lll}0 & 0 & 1\end{array}\right]^{\top}$ satisfies the restrictions given by the cone $K$. Let ${ }^{Q} v$ be the vector part of $\mathcal{V}=Q * e_{z} * Q^{*}$. Then the two restrictions are the same

$$
Q \in K \quad \Longleftrightarrow \quad Q_{v} \in K
$$

\section{Representing Sets of Orientations as Convex SETS}

The results in this section are based on the observation that a set of orientations can be represented as a convex cone. We can then use the properties of convex cones to transform the set of orientations into a different set that preserves convexity. We start by giving an alternative proof of the ZYZ-sequence $\left(\|\cdot\|_{2}\right.$-norm) presented in the previous section. This gives an intuitive understanding of why the $\|\cdot\|_{2}$-norm is so easy to work with. We then show how we can use the properties of duality of convex cones to find the cone given by the $\|\cdot\|_{\infty}$-norm from the cone given by the $\|\cdot\|_{1}$-norm.

\section{A. The 2-norm}

Given a quaternion by the ZYZ-sequence

$$
Q=Q_{z} * Q_{y} * Q_{f}
$$

where

$$
\begin{aligned}
Q & =\left[\begin{array}{llll}
q_{0} & q_{1} & q_{2} & q_{3}
\end{array}\right]^{\top}, \\
Q_{z} & =\left[\begin{array}{llll}
{ }^{z} q_{0} & 0 & 0 & { }^{z} q_{3}
\end{array}\right]^{\top}, \\
Q_{y} & =\left[\begin{array}{llll}
{ }^{y} q_{0} & 0 & { }^{y} q_{2} & 0
\end{array}\right]^{\top}, \\
Q_{f} & =\left[\begin{array}{llll}
{ }^{f} q_{0} & 0 & 0 & { }^{f} q_{3}
\end{array}\right]^{\top} .
\end{aligned}
$$

$Q$ can then be written as

$$
Q=\left[\begin{array}{l}
q_{0} \\
q_{1} \\
q_{2} \\
q_{3}
\end{array}\right]=\left[\begin{array}{ccc}
{ }^{z} q_{0}{ }^{y} q_{0}{ }^{f} q_{0}-{ }^{z} q_{3}{ }^{y} q_{0}{ }^{f} q_{3} \\
{ }^{z} q_{3}{ }^{y} q_{2}{ }^{f} q_{0}+{ }^{z} q_{0}{ }^{y} q_{2}{ }^{f} q_{3} \\
{ }^{z} q_{0}{ }^{y} q_{2}{ }^{f} q_{0}+{ }^{z} q_{3}{ }^{y} q_{2}{ }^{f} q_{3} \\
{ }^{z} q_{0}{ }^{y} q_{0}{ }^{f} q_{3}+{ }^{z} q_{3}{ }^{y} q_{0}{ }^{f} q_{0}
\end{array}\right] .
$$

From this we get the following property that we will use in the sequel

$$
\begin{aligned}
q_{1}^{2}+q_{2}^{2}= & \left({ }^{z} q_{3}{ }^{y} q_{2}{ }^{f} q_{0}+{ }^{z} q_{0}{ }^{y} q_{2}{ }^{f} q_{3}\right)^{2} \\
& +\left({ }^{z} q_{0}{ }^{y} q_{2}{ }^{f} q_{0}+{ }^{z} q_{3}{ }^{y} q_{2}{ }^{f} q_{3}\right)^{2} \\
= & { }^{z} q_{3}^{2}{ }^{y} q_{2}^{2}{ }^{f} q_{0}^{2}-2{ }^{z} q_{0}{ }^{z} q_{3}{ }^{y} q_{2}^{2}{ }^{f} q_{0}{ }^{f} q_{3}+{ }^{z} q_{0}^{2}{ }^{y} q_{2}^{2}{ }^{f} q_{3}^{2} \\
& +{ }^{z} q_{0}^{2}{ }^{y} q_{2}^{2}{ }^{f} q_{0}^{2}+2{ }^{z} q_{0}{ }^{z} q_{3}{ }^{y} q_{2}^{2}{ }^{f} q_{0}{ }^{f} q_{3}+{ }^{z} q_{3}^{2}{ }^{y} q_{2}^{2}{ }^{f} q_{3}^{2} \\
= & { }^{y} q_{2}^{2}\left({ }^{z} q_{3}^{2}\left({ }^{f} q_{0}^{2}+{ }^{f} q_{3}^{2}\right)+{ }^{z} q_{0}^{2}\left({ }^{f} q_{0}^{2}+{ }^{f} q_{3}^{2}\right)\right) \\
= & { }^{y} q_{2}^{2} .
\end{aligned}
$$

A unit vector in the direction of the $z$-axis is rotated by the quaternion $Q$ by

$$
\mathcal{V}=\left[\begin{array}{c}
v_{0} \\
v_{1} \\
v_{2} \\
v_{3}
\end{array}\right]=\left[\begin{array}{c}
0 \\
2{ }^{y} q_{0}{ }^{y} q_{2}\left({ }^{z} q_{0}^{2}-{ }^{z} q_{3}^{2}\right) \\
{ }^{y} q_{0}{ }^{y} q_{2}{ }^{z} q_{0}{ }^{z} q_{3} \\
{ }^{y} q_{0}^{2}-{ }^{y} q_{2}^{2}
\end{array}\right] .
$$

The $\|\cdot\|_{2}$-norm of the vector part of $\mathcal{V}$ can be used to restrict the rotation $\beta$ in (10) by

$$
\sqrt{v_{1}^{2}+v_{2}^{2}} \leq \nu\left|v_{3}\right|
$$

where $\nu=\tan \beta_{\text {lim }}$. We can then write the restriction as

$$
\frac{\sqrt{v_{1}^{2}+v_{2}^{2}}}{\left|v_{3}\right|} \leq \nu=\tan \beta_{l i m} .
$$


If we assume that the vector part of $\mathcal{V}$ is of unit length, the rotation $\beta$ can also be found from

$$
\begin{aligned}
\beta & =\arcsin \sqrt{v_{1}^{2}+v_{2}^{2}} \\
& =\arcsin \sqrt{\left({ }^{y} q_{0}{ }^{y} q_{2}\left({ }^{z} q_{0}^{2}-{ }^{z} q_{3}^{2}\right)\right)^{2}+\left({ }^{y} q_{0}{ }^{y} q_{2}{ }^{z} q_{0}{ }^{z} q_{3}\right)^{2}} \\
& =\arcsin \sqrt{4\left({ }^{y} q_{0}^{2}{ }^{y} q_{2}^{2}{ }^{z} q_{0}^{4}+2{ }^{y} q_{0}^{2}{ }^{y} q_{2}^{2}{ }^{z} q_{0}^{2}{ }^{z} q_{3}^{2}+{ }^{y} q_{0}^{2}{ }^{y} q_{2}^{2}{ }^{z} q_{3}^{4}\right)} \\
& =\arcsin \sqrt{{ }^{4}{ }^{y} q_{0}^{2}{ }^{y} q_{2}^{2}\left({ }^{z} q_{0}^{2}+{ }^{z} q_{3}^{2}\right)^{2}} \\
& =\arcsin \sqrt{{ }^{4}{ }^{y} q_{0}^{2}{ }^{y} q_{2}^{2}}
\end{aligned}
$$

If we use that ${ }^{y} q_{0}^{2}=\left(1-{ }^{y} q_{2}^{2}\right)$ and that $2 \arcsin x=$ $\arcsin 2 x \sqrt{1-x^{2}}$ together with the relation in (36) and $\beta \geq 0 \rightarrow{ }^{y} q_{2} \geq 0$, we get

$$
\begin{aligned}
\beta & =\arcsin \sqrt{{ }^{4}{ }^{y} q_{0}^{2}{ }^{y} q_{2}^{2}} \\
& =\arcsin \left(2{ }^{y} q_{2} \sqrt{\left(1-{ }^{y} q_{2}^{2}\right)}\right) \\
& =2 \arcsin \left({ }^{y} q_{2}\right) \\
& =2 \arcsin \sqrt{q_{1}^{2}+q_{2}^{2}} .
\end{aligned}
$$

We see that we can represent the restriction on $\beta$ in terms of $v_{1}, v_{2}$ and $v_{3}$. This example does not give us any new information, it just shows us how the problem can be formulated in a different way and that the two approaches to the problem give us the same answer. We now use this approach to restrict orientations by the 1 - and $\infty$-norms. We will see that this approach allows us to find limits on rotations that we could not find by the approaches presented in previous publications.

\section{B. The 1-norm}

The $\|\cdot\|_{1}$-cone is given by

$$
\left|v_{x}\right|+\left|v_{y}\right| \leq \mu\left|v_{z}\right|
$$

A unit vector $e_{z}=\left[\begin{array}{lll}0 & 0 & 1\end{array}\right]^{\top}$ rotated by the quaternion $Q$ is given as

$$
\left[\begin{array}{l}
v_{x} \\
v_{y} \\
v_{z}
\end{array}\right]=\left[\begin{array}{l}
2\left(q_{0} q_{2}+q_{1} q_{3}\right) \\
2\left(q_{2} q_{3}-q_{0} q_{1}\right) \\
2\left(q_{0}^{2}+q_{3}^{2}\right)-1
\end{array}\right]
$$

Thus, the restriction that the central axis lies inside the cone defined by (40) is given by

$$
\left|2\left(q_{0} q_{2}+q_{1} q_{3}\right)\right|+\left|2\left(q_{2} q_{3}-q_{0} q_{1}\right)\right| \leq \mu\left|2\left(q_{0}^{2}+q_{3}^{2}\right)-1\right| .
$$

or alternatively

$$
\left|2{ }^{y} q_{0}{ }^{y} q_{2}\right|+\left|2{ }^{x} q_{0}{ }^{x} q_{1}\left({ }^{y} q_{2}^{2}-{ }^{y} q_{0}^{2}\right)\right| \leq \mu\left|\left({ }^{x} q_{1}^{2}-{ }^{x} q_{0}^{2}\right)\left({ }^{y} q_{2}^{2}-{ }^{y} q_{0}^{2}\right)\right| .
$$

\section{The $\infty$-norm}

In the following we will use that the $\|\cdot\|_{\infty}$-cone is the dual of the $\|\cdot\|_{1}$-cone to remove the non-differentiability from the restriction on the $\|\cdot\|_{\infty}$-norm.

Proposition 3.1: Let the vector part of the quaternion $\mathcal{V}=$ $Q * e_{z} * Q^{*}$ be denoted $v_{Q}=\left[\begin{array}{lll}Q_{v_{x}} & Q_{v_{y}} & Q_{v_{z}}\end{array}\right]^{\top}$ and the vector part of the quaternion $\mathcal{V}=P * e_{z} * P^{*}$ be denoted
$v_{P}=\left[\begin{array}{lll}{ }^{P} v_{x} & P_{v_{y}} & { }^{P} v_{z}\end{array}\right]^{\top}$. Further let $K^{*}$ be the dual of $K$ and $Q \in K$ where $K$ is the cone defined by the $\|\cdot\|_{1}$-norm

$$
\left|{ }^{Q} v_{x}\right|+\left|{ }^{Q} v_{y}\right| \leq\left.\mu\right|^{Q} v_{z} \mid
$$

for some $\mu$. Then, for some $\eta$, the two restrictions

$$
\begin{gathered}
Q_{v_{x}} P_{v_{x}}+{ }^{Q} v_{y}{ }^{P} v_{y}+{ }^{Q} v_{z}{ }^{P} v_{z} \geq 0 \\
\mathbb{1} \\
\max \left\{{ }^{P} v_{x},{ }^{P} v_{y}\right\} \leq\left.\eta\right|^{P} v_{z} \mid
\end{gathered}
$$

both guarantee that $P \in K^{*}$.

Proof: The proof follows directly from the definition of dual cones in (13) which we rewrite as

$$
\begin{aligned}
K^{*} & =\left\{v_{P} \mid v_{Q}^{\top} v_{P} \geq 0, \forall v_{Q} \in K\right\} \\
& =\left\{P \mid v_{Q}^{\top} v_{P} \geq 0, \forall v_{Q} \in K\right\} .
\end{aligned}
$$

We now need to determine how to choose $\mu$ so that the $\|\cdot\|_{\infty}$ cone allows the desired maximum rotation. We will denote the $\|\cdot\|_{\infty}$-cone and the $\|\cdot\|_{1}$-cone equivalent if they allow the same maximum rotation, i.e. the maximum allowed rotation for the $\|\cdot\|_{1}$-cone in the direction of one of the principal axes is the same as the maximum allowed rotation for the $\|\cdot\|_{\infty}$-cone when $x= \pm y$.

Proposition 3.2: Given a cone $K$ defined by

$$
\left|{ }^{Q} v_{x}\right|+\left|{ }^{Q} v_{y}\right| \leq\left.\mu\right|^{Q} v_{z} \mid
$$

and a dual cone $K^{*}$ defined by

$$
\max \left\{{ }^{P} v_{x},{ }^{P} v_{y}\right\} \leq\left.\eta\right|^{P} v_{z} \mid .
$$

Then $K$ and $K^{*}$ are equivalent if

$$
\mu=\sqrt{2} \tan \left(\frac{\pi}{2}-\arctan \nu\right) .
$$

Proof: We show the specific proof for the two cones in question. We do this by showing that the proposition is true for the limit points of the set. As the set is closed and convex, we conclude that the set defined by these limit points is the desired set.

For the principal axes the maximum allowed rotations are $\mu$ and $\nu$ for the $\|\cdot\|_{1}$-cone and the $\|\cdot\|_{\infty}$-cone respectively (Equations (28) and (21)). When $x= \pm y$ the maximum allowed rotations are $\frac{\mu}{\sqrt{2}}$ (Equation (29)) for the $\|\cdot\|_{1}$-cone and $\sqrt{2} \nu$ (Equation (22)) for the $\|\cdot\|_{\infty}$-cone:

\begin{tabular}{l|c|c} 
& $\|\cdot\|_{1}$-cone & $\|\cdot\|_{\infty}$-cone \\
\hline Principal axes & $\mu$ & $\nu$ \\
\hline$x= \pm y$ & $\frac{\mu}{\sqrt{2}}$ & $\sqrt{2} \nu$
\end{tabular}

Let $\alpha=\arctan \nu$ and $\beta=\arctan \mu$. By definition the limit points for the two cones are orthogonal (using equality in (13)), so we have that $\beta=\frac{\pi}{2}-\alpha$ and thus

$$
\mu=\tan \left(\frac{\pi}{2}-\arctan \nu\right)
$$

Thus, a rotation $\alpha=\arctan \nu$ for the $\|\cdot\|_{\infty}$-cone corresponds to a rotation $\beta=\arctan \mu$ for the $\|\cdot\|_{1}$-cone for the principal axes (row one above). This means that if we choose $\mu=\nu$ 
we will allow the same orientation error in the direction of the principal axes for both cones. When $x= \pm y$ we have

$$
\begin{aligned}
\tan \left(\frac{\pi}{2}-\arctan \sqrt{2} \nu\right) & =\frac{1}{\sqrt{2}} \tan \left(\frac{\pi}{2}-\arctan \nu\right) \\
& =\frac{1}{\sqrt{2}} \mu
\end{aligned}
$$

So that $\sqrt{2} \nu$ corresponds to $\frac{\mu}{\sqrt{2}}$ for $x= \pm y$ (the second row). This means that if we choose $\mu=\nu$ we will allow twice as big orientation error for the $\|\cdot\|_{\infty}$-cone than for the $\|\cdot\|_{1}$-cone in the direction of $x= \pm y$.

Finally we scale the $\|\cdot\|_{1}$-cone by $\sqrt{2}$ to get the same maximum rotation for both cones

\begin{tabular}{l|c|c} 
& $\|\cdot\|_{1}$-cone & $\|\cdot\|_{\infty}$-cone \\
\hline Principal axes & $\sqrt{2} \mu$ & $\nu$ \\
\hline$x= \pm y$ & $\mu$ & $\sqrt{2} \nu$
\end{tabular}

which concludes the proof.

Note that for the $\|\cdot\|_{1}$-cone the maximum allowed rotation is around the principal axes, while for the $\|\cdot\|_{\infty}$-cone it is around the axes defined by $x= \pm y$. The geometric interpretation of the proof can be visualised by looking at Figure 1.

The approach given by Proposition 3.2 is rather theoretical and the transformation from the $\|\cdot\|_{1}$-cone to the $\|\cdot\|_{\infty}$-cone may not be very easy in practice. However, we can use Equation (49) in Proposition 3.2 to find an easy transformation from the $\|\cdot\|_{1}$-cone to the $\|\cdot\|_{\infty}$-cone. As the $\|\cdot\|_{\infty}$-cone only takes into account the maximum rotation about the $x$ - or $y$ axis we can cancel the other rotation from the restrictions on the $\|\cdot\|_{1}$-cone in (42) and (43).

The restriction on the orientation about the $x$-axis is given by

$$
\begin{gathered}
\left|2\left(q_{2} q_{3}-q_{0} q_{1}\right)\right| \leq \nu\left|2\left(q_{0}^{2}+q_{3}^{2}\right)-1\right|, \\
\left|2^{x} q_{0}{ }^{x} q_{1}\right| \leq \nu\left|\left({ }^{x} q_{1}^{2}-{ }^{x} q_{0}^{2}\right)\right|,
\end{gathered}
$$

and the restriction on the orientation about the $y$-axis is given by

$$
\begin{gathered}
\left|2\left(q_{0} q_{2}+q_{1} q_{3}\right)\right| \leq \nu\left|2\left(q_{0}^{2}+q_{3}^{2}\right)-1\right|, \\
\left|2{ }^{y} q_{0}{ }^{y} q_{2}\right| \leq \nu\left|\left({ }^{x} q_{1}^{2}-{ }^{x} q_{0}^{2}\right)\left({ }^{y} q_{2}^{2}-{ }^{y} q_{0}^{2}\right)\right|,
\end{gathered}
$$

which guarantees that $Q \in K^{*} . \mu$ is the maximum rotation as defined by the $\|\cdot\|_{1}$-cone. Thus to allow the same maximum rotation for the $\|\cdot\|_{\infty}$-cone we define $\mu$ as in Equation (49) and get

$$
\begin{aligned}
& \left|2\left(q_{2} q_{3}-q_{0} q_{1}\right)\right| \leq \mu_{x}\left|2\left(q_{0}^{2}+q_{3}^{2}\right)-1\right|, \\
& \left|2\left(q_{0} q_{2}+q_{1} q_{3}\right)\right| \leq \mu_{y}\left|2\left(q_{0}^{2}+q_{3}^{2}\right)-1\right|,
\end{aligned}
$$

which then defines the equivalent $\|\cdot\|_{\infty}$-cone.

Note that we can choose $\mu_{x}$ and $\mu_{y}$ differently so that we can allow different orientation errors around different axes. Note also that these constraints are exact. This was not the case for the orientation around the $x$ - and $y$-axes presented in [3].

\section{Conclusions}

The formalism for presenting sets of orientations as convex cones has been derived. Three different sets of orientations that can easily be described by simple constraints in quaternion space have been presented. The properties of dual convex cones are used to fine simple representations for the sets of orientations and computationally faster and more accurate tests to verify if a quaternion lies within the given set. By representing the sets of orientations as dual convex cones the non-differentiability properties that arise when applying the definition of the desired cones directly are eliminated.

\section{ACKNOWLEDGMENT}

The authors wish to acknowledge the support of the Norwegian Research Council and the TAIL IO project for their continued funding and support for this research. The TAIL IO project is an international cooperative research project led by StatoilHydro and an R\&D consortium consisting of ABB, IBM, Aker Kvaerner and SKF. During the work with this paper the first author was with University of California at Berkeley.

\section{REFERENCES}

[1] V. Potkonjak, G. Dordevic, D. Kostic and M. Rasic, Dynamics of anthropomorphic painting robot: Quality analysis and cost reduction Robotics and Autonomous Systems, Vol. 32, No 1, 2000.

[2] P. J. From and J. T. Gravdahl, General solutions to Kinematic and Functional Redundancy Proc. 46th IEEE Conf. on Decision and Control, 2007.

[3] P. J. From and J. T. Gravdahl, On the Equivalence of Orientation Error and Positive Definiteness of Matrices Proc. 10th International Conference on Control, Automation and Vision, 2008.

[4] B. Alpern, L. Carter and M. Grayson and C. Pelkie, Orientation Maps: Techniques for Visualizing Rotations (A Consumers Guide) IEEE Conference on Visualization, 1993.

[5] J. B. Kuipers, Quaternions and Rotation Sequences Princeton University Press, 2002.

[6] J. M. Ahuactzin and K. K. Gupka, The Kinematic Roadmap: A Motion Planning Based Global Approach for Inverse Kinematics of Redundant Robots IEEE Trans. on Robotics and Automation, 1999.

[7] L. Vandenberghe, S Boyd and S. P. Wu, Determinant maximization with linear matrix inequality constraints http://stanford.edu/ boyd/papers/maxdet.html, 1996.

[8] P. J. From and J. T. Gravdahl, Representing Attitudes as Sets of Frames Proc. American Control Conference, 2007. 